\title{
Fault diagnoser design for untimed continuous Petri nets
}

\author{
Carla Seatzu, Cristian Mahulea, Maria Paola Cabasino, Manuel Silva
}

\begin{abstract}
Continuous Petri nets can be viewed as an approximation of the classical discrete models introduced to cope with the state explosion problem typical of discrete event systems. In this paper we consider free-labeled Petri net systems, and assume that certain transitions, including all those modeling faulty behaviors, are unobservable, i.e., they are labeled with the empty word.

Based on the notion and characterization of the set of justifications of a given observation (and the corresponding set of $j$-vectors), we provide a diagnosis approach using the solution of a certain number of linear programming problems.
\end{abstract}

\section{INTRODUCTION}

The problem of fault diagnosis is clearly a main issue in most of the engineering applications because of the practical need of ensuring the correct and safe functioning of systems. This is the reason why it has been so extensively investigated in the last decades. Most of the results have been developed within the framework of time-driven systems, but several significant results have also been proposed for discrete event systems. In particular, a series of original theoretical approaches have been proposed using automata, e.g., by Boel and van Schuppen [1], by Debouk et al. [2], by Hashtrudi Zad et al. [3], by Jiang and Kumar [4], by Lunze and Schroder [5], and by Sampath et al. [6], [7].

Petri net (PN) models have also been used in this context: the intrinsically distributed nature of PNs, where the notion of state (i.e., marking) and action (i.e., transition) is local, has often been an asset to reduce the computational complexity involved in solving a diagnosis problem. Among the different contributions in this area we recall the work of Ushio et al. [8], Benveniste et al. [9], [10], Jiroveanu and Boel [11], Giua and Seatzu [12], and Cabasino et al. [13].

Recently, a particular hybrid model based on PNs has received some attention. This model is called continuous Petri net (contPN) [14], [15]. It can be seen as a relaxation of PNs where the constraint that markings and transitions firings are integer is removed.

In this paper we focus on the problem of designing a diagnoser for untimed contPNs, thus we can assume that the net behavior is asynchronous and sequential. We also assume that the net structure is known, as well as the initial marking.

This work has been partially supported by the European Community's Seventh Framework Programme under project DISC (Grant Agreement n. INFSO-ICT-224498). This work was partially supported by the project CICYT - FEDER DPI2006-15390 at the University of Zaragoza.

C. Seatzu and M.P. Cabasino are with the Department of Electrical and Electronic Engineering, University of Cagliari, Piazza D'Armi, 09123 Cagliari, Italy \{seatzu,cabasino@diee.unica.it\}.

C. Mahulea, and M. Silva are with the Department of Computer Science and System Engineering, University of Zaragoza, Maria de Luna 1, 50018 Zaragoza, Spain \{cmahulea, silva@unizar.es\}.
The set of transitions is partitioned into two subsets: observable and unobservable transitions. Unobservable transitions may either model regular behavior or fault behavior, while fault transitions are partitioned into different fault classes. After each observable transition fires we observe its firing quantity, which is the continuous counterpart of the number of firings of each transition. Our goal is that of establishing if some transition in a given fault class may have fired, given the actual observation. In particular, based on the results in [13], we define four fault diagnosis states that correspond to four different degrees of alarm. The diagnoser is a function that associates to each fault class and each observation a diagnosis state.

We prove that, under certain assumptions on the unobservable subnet, the set of j-vectors, that keep track of the set of unobservable transitions that may have fired to enable the current observation, is convex. In particular, we prove that in such a case the diagnosis problem can be written in terms of linear programming problems (LPPs). This is a significant advantage with respect to discrete PNs. In fact, in such a case the computation of the diagnosis states requires the solution of linear integer programming problems.

\section{BACKGROUND ON UNTIMED CONTPNS}

In this section we provide the basic background on untimed contPNs.

Definition 1: A contPN system is a pair $\left\langle\mathcal{N}, \boldsymbol{m}_{0}\right\rangle$, where:

- $\mathcal{N}=\langle P, T$, Pre, Post $\rangle$ is the net structure with two disjoint sets of places $P$ and transitions $T$; pre and post incidence matrices Pre, Post $\in \mathbb{R}_{\geq 0}^{|P| \times|T|}$, denote respectively the weight of the arcs from places to transitions and from transitions to places;

- $\boldsymbol{m}_{0} \in \mathbb{R}_{>0}^{|P|}$ is the initial marking.

We denote as $m=|P|$ and $n=|T|$ the cardinality of the set of places and transitions, respectively.

The input and output set of a node $x \in P \cup T$ is denoted by ${ }^{\bullet} x$ and $x^{\bullet}$, respectively. The token load of a place $p_{i}$ at the marking $\boldsymbol{m}$ is denoted by $\boldsymbol{m}\left(p_{i}\right)$ or simply by $m_{i}$.

A transition $t_{j} \in T$ is enabled at a marking $\boldsymbol{m}$ iff $\forall p_{i} \in{ }^{\bullet} t_{j}, \boldsymbol{m}\left(p_{i}\right) \geq 0$ and the enabling degree of $t_{j}$ at $\boldsymbol{m}$ is $\operatorname{enab}\left(t_{j}, \boldsymbol{m}\right)=\min _{p_{i} \in t_{j}} \frac{m_{i}}{\boldsymbol{P r e}\left(p_{i}, t_{j}\right)}$.

When a transition $t_{j}$ is enabled at a marking $m$ it can be fired. The main difference with respect to (w.r.t.) discrete PNs is that in the case of contPNs it can be fired in any real amount $\alpha$, with $0 \leq \alpha \leq \operatorname{enab}\left(t_{j}, \boldsymbol{m}\right)$ and it is not limited only to a natural number. Such a firing yields to a new marking $\boldsymbol{m}^{\prime}=\boldsymbol{m}+\alpha \cdot \boldsymbol{C}\left(\cdot, t_{j}\right)$, where $\boldsymbol{C}=\boldsymbol{P o s t}-$ Pre is the token flow matrix (or incidence matrix). This firing is also denoted $\boldsymbol{m}\left[t_{j}(\alpha)\right\rangle \boldsymbol{m}^{\prime}$. 
If a marking $\boldsymbol{m}$ is reachable from the initial marking through a firing sequence $\sigma=t_{r 1}\left(\alpha_{1}\right) t_{r 2}\left(\alpha_{2}\right) \cdots t_{r k}\left(\alpha_{k}\right)$, and we denote by $\sigma \in \mathbb{R}_{>0}^{|T|}$ the firing count vector whose component associated to a transition $t_{j}$ is $\sigma_{j}=\sum_{h \in H\left(\sigma, t_{j}\right)} \alpha_{h}$, where $H\left(\sigma, t_{j}\right)=\left\{h=1, \ldots, k \mid t_{r_{h}}=t_{j}\right\}$, then we can write $\boldsymbol{m}=\boldsymbol{m}_{0}+\boldsymbol{C} \cdot \boldsymbol{\sigma}$, which is called the fundamental equation or state equation.

The set of all fireable sequences is $\mathcal{L}\left(\mathcal{N}, \boldsymbol{m}_{0}\right)$, while the set of all markings that are reachable with a finite firing sequence is denoted by $R S^{u t}\left(\mathcal{N}, \boldsymbol{m}_{0}\right)$. An interesting property of $R S^{u t}\left(\mathcal{N}, \boldsymbol{m}_{0}\right)$ is that it is a convex set [16]. That is, if two markings $\boldsymbol{m}_{1}$ and $\boldsymbol{m}_{2}$ are reachable, then any marking $\boldsymbol{m}_{3}=\alpha \cdot \boldsymbol{m}_{1}+(1-\alpha) \cdot \boldsymbol{m}_{2}, \forall \alpha \in[0,1]$ is also reachable.

The net $\mathcal{N}$ is consistent iff $\exists \boldsymbol{x}>0$ such that $\boldsymbol{C} \cdot \boldsymbol{x}=\mathbf{0}$.

A PN $\mathcal{N}=\langle P, T$, Pre, Post $\rangle$ is a state machine if $\forall t \in$ $T,|\bullet t|=\left|t^{\bullet}\right| \leq 1$ and $\operatorname{Pre}(p, t), \operatorname{Post}(p, t) \in\{0,1\}$ for any $p \in P$ and any $t \in T$.

Given a net $\mathcal{N}=\langle P, T, \boldsymbol{P r}$, Post $\rangle$, and a subset $T^{\prime} \subseteq T$ of its transitions, the $T^{\prime}$-induced subnet of $\mathcal{N}$ is the new net $\mathcal{N}^{\prime}=\left\langle P, T^{\prime}, \boldsymbol{P r} \boldsymbol{e}^{\prime}, \boldsymbol{P o s t}^{\prime}\right\rangle$ where $\boldsymbol{P r} \boldsymbol{e}^{\prime}, \boldsymbol{P o s t}^{\prime}$ are the restriction of Pre, Post to $T^{\prime}$. The net $\mathcal{N}^{\prime}$ can be thought as obtained from $\mathcal{N}$ removing all transitions in $T \backslash T^{\prime}$. We also write $\mathcal{N}^{\prime} \prec_{T^{\prime}} \mathcal{N}$.

Given a subset $T^{\prime} \subseteq T$, the projection $\Pi$ of a sequence $\sigma \in T^{*}$ over $T^{\prime}$ is defined as $\Pi: T^{*} \rightarrow T^{\prime *}$ such that: (i) $\Pi(\varepsilon)=\varepsilon$, where $\varepsilon$ denotes the empty word; (ii) for all $\sigma \in T^{*}$ and $t \in T, \Pi(\sigma t)=\Pi(\sigma) t$ if $t \in T^{\prime}$, and $\Pi(\sigma t)=\Pi(\sigma)$ otherwise. Here $T^{*}$ denotes the set of all possible sequences obtainable combining elements in $T$, included the empty word.

Given a sequence $\sigma \in \mathcal{L}\left(\mathcal{N}, \boldsymbol{m}_{0}\right)$, we denote $w=\Pi_{o}(\sigma)$ the corresponding observed word.

In the following, with a little abuse of notation, we will write that $w \in T_{o}^{*}$.

\section{EXPLANATIONS AND E-VECTORS}

In this section we propose a procedure to design a diagnoser for contPNs based on the following three assumptions.

(A1) The initial marking of the net is known.

(A2) The set of transitions is partitioned as $T=T_{o} \cup T_{u}$ where $T_{o}$ is the set of observable transitions, and $T_{u}$ is the set of unobservable transitions.

(A3) The $T_{u}$-induced net has no spurious solutions.

In simple words, the third assumption implies that all markings $\boldsymbol{m} \in \mathbb{R}_{\geq 0}^{m}$ such that $\boldsymbol{m}=\boldsymbol{m}_{0}+C \cdot \boldsymbol{\sigma}$, with $\boldsymbol{\sigma} \geq \mathbf{0}$, are reachable.

Proposition 2: Let $\left\langle\mathcal{N}, \boldsymbol{m}_{0}\right\rangle$ be a contPN system. All markings $\boldsymbol{m} \in \mathbb{R}_{\geq 0}^{m}: \boldsymbol{m}=\boldsymbol{m}_{0}+C \cdot \boldsymbol{\sigma}$, with $\boldsymbol{\sigma} \geq \mathbf{0}$, are reachable, i.e., $\mathcal{N}$ has no spurious solutions, if at least one of the following two conditions is satisfied:

- $\mathcal{N}$ is acyclic [17];

- $\mathcal{N}$ is consistent and all transitions are firable from $\boldsymbol{m}_{0}$ [16].
Definition 3: Given a set of markings $\mathcal{M}$ and an observable transition $t \in T_{o}$ firing an amount $\alpha$, we define

$$
\begin{aligned}
\Sigma(\mathcal{M}, t(\alpha))=\left\{\sigma \in T_{u}^{*} \mid\right. & \exists \boldsymbol{m} \in \mathcal{M}: \\
& \left.\boldsymbol{m}[\sigma\rangle \boldsymbol{m}^{\prime}, \boldsymbol{m}^{\prime} \geq \alpha \cdot \operatorname{Pre}(\cdot, t)\right\}
\end{aligned}
$$

the set of explanations of $t(\alpha)$ at markings in $\mathcal{M}$, and we define

$$
Y(\mathcal{M}, t(\alpha))=\left\{\boldsymbol{e} \in \mathbb{R}_{\geq 0}^{n_{u}} \mid \exists \sigma \in \Sigma(\mathcal{M}, t(\alpha)): \boldsymbol{\sigma}=\boldsymbol{e}\right\}
$$

the $e$-vectors (or explanation vectors) of $t(\alpha)$ at $\mathcal{M}$, i.e., the firing vectors associated to the explanations.

Thus, if $\mathcal{M}$ is the set of markings in which the system is known to be, e.g., the set of markings that are consistent with an observed word, and $t(\alpha)$ is a new observation, then $\Sigma(\mathcal{M}, t(\alpha))$ coincides with the set of unobservable sequences whose firing may have enabled the firing of $t(\alpha)$ starting from any marking in $\mathcal{M}$.

The following proposition shows a nice feature of $Y(\mathcal{M}, t(\alpha))$.

Proposition 4: Given a convex set of markings $\mathcal{M}$ and an observable transition $t \in T_{o}$ firing an amount $\alpha$, under assumption (A3), the set $Y(\mathcal{M}, t(\alpha))$ is convex.

Proof: To prove this we show that, given an arbitrary couple of vectors $\boldsymbol{y}_{1}, \boldsymbol{y}_{2} \in Y(\mathcal{M}, t(\alpha))$, any convex combination of them belongs to the set $Y(\mathcal{M}, t(\alpha))$.

If $\boldsymbol{y}_{1}, \boldsymbol{y}_{2}$ are e-vectors of $t(\alpha)$ at $\mathcal{M}$, it means that there exist two markings $\boldsymbol{m}_{1}^{\prime}, \boldsymbol{m}_{2}^{\prime} \in \mathcal{M}$ such that

$$
\begin{aligned}
& \boldsymbol{m}_{1}=\boldsymbol{m}_{1}^{\prime}+C \cdot \boldsymbol{y}_{1} \geq \alpha \cdot \operatorname{Pre}(\cdot, t), \\
& \boldsymbol{m}_{2}=\boldsymbol{m}_{2}^{\prime}+C \cdot \boldsymbol{y}_{2} \geq \alpha \cdot \operatorname{Pre}(\cdot, t) .
\end{aligned}
$$

Now, let $\tilde{\boldsymbol{y}}=\delta \cdot \boldsymbol{y}_{1}+\beta \cdot \boldsymbol{y}_{2}$ where $\delta, \beta \in[0,1]$ and $\delta+\beta=1$. Being the net system continuous, by assumption (A3) there exists a firing sequence $\tilde{\sigma}$ with $\tilde{\boldsymbol{\sigma}}=\tilde{\boldsymbol{y}}$ such that $\tilde{\sigma}$ is enabled at $\boldsymbol{m}^{\prime}=\delta \cdot \boldsymbol{m}_{1}^{\prime}+\beta \cdot \boldsymbol{m}_{2}^{\prime} \in \mathcal{M}$. But,

$$
\begin{aligned}
\boldsymbol{m} & =\boldsymbol{m}^{\prime}+C \cdot \tilde{\boldsymbol{y}} \\
& =\delta \cdot \boldsymbol{m}_{1}^{\prime}+\beta \cdot \boldsymbol{m}_{2}^{\prime}+\delta \cdot C \cdot \boldsymbol{y}_{1}+\beta \cdot C \cdot \boldsymbol{y}_{2} \\
& =\delta \cdot \boldsymbol{m}_{1}+\beta \cdot \boldsymbol{m}_{2} \\
& \geq \delta \cdot \alpha \cdot \operatorname{Pre}(\cdot, t)+\beta \cdot \alpha \cdot \operatorname{Pre}(\cdot, t) \\
& =\alpha \cdot \operatorname{Pre}(\cdot, t)
\end{aligned}
$$

thus proving the statement.

If the net system is bounded the set $Y(\mathcal{M}, t(\alpha))$ can be easily characterized in linear algebraic terms using the following algorithm that assumes that the set $\mathcal{M}$ is convex and defined as (see the following Remark 6):

$$
\mathcal{M}=\left\{\left[\begin{array}{l}
\boldsymbol{m} \\
\boldsymbol{\sigma}_{u}
\end{array}\right] \in \mathbb{R}_{\geq 0}^{m+n_{u}} \mid A_{\mathcal{M}} \cdot\left[\begin{array}{l}
\boldsymbol{m} \\
\boldsymbol{\sigma}_{u}
\end{array}\right] \leq b_{\mathcal{M}}\right\} .
$$

Note that this is exactly the case when the set $\mathcal{M}$ coincides with the set of markings consistent with the sequence $v$ of observations before $t(\alpha)$, that we denoted as $\mathcal{C}(v)$, namely with the set of markings in which the system may be given the observation $v$. We recently proved the convexity of $\mathcal{C}(v)$ in [18] where we also provided an algorithm to express it in the form of eq. (2).

Algorithm 5 (Computation of $Y(\mathcal{M}, t(\alpha))$ ):

1) Compute the set of vertices $\mathcal{E}_{\mathcal{M}}$ of $\mathcal{M}$. 
2) Let $E=\emptyset$.

3) For all $\boldsymbol{e}_{i}=\left[\begin{array}{ll}\tilde{\boldsymbol{m}}^{T} & \tilde{\boldsymbol{\sigma}}_{\boldsymbol{u}}^{T}\end{array}\right]^{T} \in \mathcal{E}_{\mathcal{M}}$ :

a) compute the set of vertices $E_{i}=\left[\begin{array}{ll}\boldsymbol{m}^{T} & \boldsymbol{\sigma}_{u}^{T}\end{array}\right]^{T}$ of the polytope ${ }^{1}$ defined as

$$
\left\{\begin{array}{l}
\boldsymbol{m}=\tilde{\boldsymbol{m}}+C_{u} \cdot \boldsymbol{\sigma}_{u} \\
\boldsymbol{m} \geq \alpha \cdot \operatorname{Pre}(\cdot, t) \\
\boldsymbol{\sigma}_{u} \geq \mathbf{0}
\end{array}\right.
$$

b) let $E=E \cup E_{i}$.

4) Let $Y(\mathcal{M}, t(\alpha))$ be the convex hull of $E$.

In simple words Algorithm 5 first computes the set of vertices of $\mathcal{M}$, denoted as $\mathcal{E}_{\mathcal{M}}$. Then, for each vertex $\boldsymbol{e}_{i}=$ $\left[\begin{array}{ll}\tilde{\boldsymbol{m}}^{T} & \tilde{\boldsymbol{\sigma}}_{\boldsymbol{u}}^{T}\end{array}\right]^{T} \in \mathcal{E}_{\mathcal{M}}$, it defines the set of markings - firing vectors $\sigma_{u}$ that can be obtained from $\tilde{\boldsymbol{m}}$ firing a sequence of unobservable transitions, with firing vector $\sigma_{u}$, that enables $t(\alpha)$. Note that by Assumption (A3) this does not lead to spurious solutions. Then the algorithm computes the set of vertices $E$ of such a set. Finally, $Y(\mathcal{M}, t(\alpha))$ is the convex hull of the union of the vertices thus obtained.

Remark 6: The set $Y(\mathcal{M}, t(\alpha))$ resulting from Algorithm 5 is defined in the $\left(m+n_{u}\right)$-dimensional space, while all vectors in $Y(\mathcal{M}, t(\alpha))$ obviously belong to the $n_{u}$-dimensional space. Analogously, the set of markings $\mathcal{M}$ defined as in eq. (2) is a subspace of $\mathbb{R}_{>0}^{m+n_{u}}$ while each marking in it belongs to $\mathbb{R}_{>0}^{m}$. Therefore, it would have been more appropriate to use different notations to distinguish $Y(\mathcal{M}, t(\alpha))$ and $\mathcal{M}$ and their definitions in the $\mathbb{R}_{>0}^{m+n_{u}}$ space. To simplify the notation we preferred not to do that when this does not introduce ambiguity.

The same remark will apply in the following to the set $Y_{\min }\left(\boldsymbol{m}_{0}, w\right)$.

\section{Minimal explanations and minimal e-Vectors}

The set of explanations can be restricted only considering the explanations with minimal firing vector. As it will be clarified in the following section, this is extremely useful when performing on-line diagnosis.

Definition 7: Given a set of markings $\mathcal{M}$ and an observable transition $t \in T_{o}$ firing an amount $\alpha$, we define

$$
\Sigma_{\text {min }}(\mathcal{M}, t(\alpha))=\left\{\begin{array}{l}
\sigma \in \Sigma(\mathcal{M}, t(\alpha)) \mid \\
\left.\quad \nexists \sigma^{\prime} \in \Sigma(\mathcal{M}, t(\alpha)): \sigma^{\prime} \leq \boldsymbol{\sigma}\right\}
\end{array}\right.
$$

the set of minimal explanations of $t(\alpha)$ at markings in $\mathcal{M}$, and we denote

$$
\begin{array}{lll}
Y_{\text {min }}(\mathcal{M}, t(\alpha))=\left\{\boldsymbol{e} \in \mathbb{R}_{\geq 0}^{n_{u}} \mid\right. & \exists \sigma \in \Sigma_{\min }(\mathcal{M}, t(\alpha)): \\
& \boldsymbol{\sigma}=\boldsymbol{e}\}
\end{array}
$$

the minimal e-vectors (or minimal explanation vectors) of $t(\alpha)$ at $\mathcal{M}$, i.e., the firing vectors associated to the minimal explanations.

In the discrete case it is proved that the set of minimal explanations is always finite provided that the set $\mathcal{M}$ is finite, thus it can be exhaustively enumerated. On the contrary, in

\footnotetext{
${ }^{1}$ A bounded polyhedron $\mathcal{P} \subset \mathbb{R}^{n}, \mathcal{P}=\left\{\boldsymbol{x} \in \mathbb{R}^{n} \quad \mid \boldsymbol{A x} \leq \boldsymbol{B}\right\}$ is called a polytope.
}

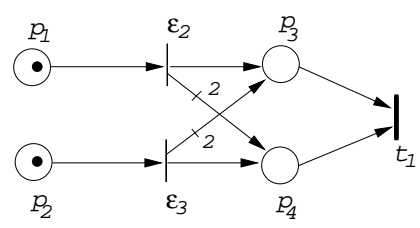

Fig. 1. The Petri net of Example 8, where $\varepsilon_{2}$ and $\varepsilon_{3}$ are not observable.

the case of contPNs, the number of minimal explanations is in general not finite. Moreover, it cannot always be characterized in linear algebraic terms. In fact, as shown by the following example, it may be a non convex set.

Example 8: Let us consider the contPN system in Fig. 1 and the observation $w=t_{1}(1)$. The following two vectors are minimal explanations for $w=t_{1}(1): \boldsymbol{\sigma}_{u}^{\prime}=\left[\begin{array}{ll}1 & 0\end{array}\right]^{T}, \boldsymbol{\sigma}_{u}^{\prime \prime}=$ $\left[\begin{array}{ll}0 & 1\end{array}\right]^{T}$.

In particular, firing $\boldsymbol{\sigma}_{u}^{\prime}$, marking $\boldsymbol{m}^{\prime}=\left[\begin{array}{llll}1 & 1 & 0 & 0\end{array}\right]^{T}+\boldsymbol{C}_{u}$. $\boldsymbol{\sigma}_{u}^{\prime}=\left[\begin{array}{lll}0 & 1 & 1\end{array}\right]^{T}$ is obtained, while firing $\boldsymbol{\sigma}_{u}^{\prime \prime}$ we get: $\boldsymbol{m}^{\prime \prime}=$ $\left[\begin{array}{llll}1 & 1 & 0 & 0\end{array}\right]^{T}+\boldsymbol{C}_{u} \cdot \boldsymbol{\sigma}_{u}^{\prime \prime}=\left[\begin{array}{llll}1 & 0 & 2 & 1\end{array}\right]^{T}$.

Note that $m_{3}^{\prime}=1$, i.e., the content of $p_{3}$ is exactly what is needed for the firing of $t_{1}(1)$; analogously, $m_{4}^{\prime \prime}=1$, i.e., the content of $p_{4}$ is the minimum marking that allows the same firing.

Now, let us consider the sequence $\sigma_{u}=\frac{1}{2} \cdot \boldsymbol{\sigma}_{u}^{\prime}+\frac{1}{2}$. $\sigma_{u}^{\prime \prime}=\left[\begin{array}{ll}\frac{1}{2} & \frac{1}{2}\end{array}\right]^{T}$. It is easy to verify that it is an explanation of $t_{1}(1)$ at $\boldsymbol{m}_{0}$, but it is not minimal. If fact, if we consider $\tilde{\boldsymbol{\sigma}}_{u}=\left[\begin{array}{ll}\frac{1}{3} & \frac{1}{3}\end{array}\right]^{T}$ it is an explanation as well, but $\tilde{\boldsymbol{\sigma}}_{u}<\boldsymbol{\sigma}_{u}$.

Even if the set $Y_{\min }(\mathcal{M}, t(\alpha))$ is not convex in general, we have been able to determine some classes of PNs for which it is convex.

Proposition 9: Let $\left\langle\mathcal{N}, \boldsymbol{m}_{0}\right\rangle$ be a contPN and $t \in T_{o}$ be a transition firing in a finite amount $\alpha$. If (A3) holds and the $T_{u}$-induced subnet is either a backward conflict-free net ${ }^{2}$ or a state machine, then the set $Y_{\min }(\mathcal{M}, t(\alpha))$ is convex.

Proof: If the $T_{u}$-induced subnet is backward conflictfree, it is proved in the discrete PN framework that the set of basic markings is a singleton [19] implying that the set of minimal explanations is a singleton as well. Exactly the same reasoning can be applied to the continuous backward conflict-free nets thus proving the statement.

If the $T_{u}$-induced subnet is a state machine, each transition has only one input and one output place. Moreover, it is an ordinary net, i.e., the weights of the arcs are unitary, hence firing an unobservable transition in an amount $\alpha$, exactly $\alpha$ tokens are removed from the input place and $\alpha$ tokens are put in the output place. Obviously, the minimal e-vectors in eq. (1) are such that those equations are satisfied as equalities. Following the same steps of Proposition 4, all inequalities are satisfied as equalities. Hence the conclusion is true.

Under the assumption of Proposition 9, Algorithm 5 can be updated as follows to compute $Y_{\min }(\mathcal{M}, t(\alpha))$.

Algorithm 10 (Computation of $Y_{\min }(\mathcal{M}, t(\alpha))$ ):

${ }^{2} \mathrm{~A} \mathrm{P} / \mathrm{T}$ net is backward conflict-free if $\forall p \in P\left|{ }^{\bullet} p\right| \leq 1$, i.e., if each place has at most one input transition. 
1) Compute the set of vertices $\mathcal{E}_{\mathcal{M}}$ of $\mathcal{M}$.

2) Let $E=\emptyset$.

3) For all $\boldsymbol{e}_{i}=\left[\begin{array}{ll}\tilde{\boldsymbol{m}}^{T} & \tilde{\boldsymbol{\sigma}}_{u}^{T}\end{array}\right] \in \mathcal{E}_{\mathcal{M}}$ :

a) compute the set of vertices $E_{i}=\left[\boldsymbol{m}^{T} \boldsymbol{\sigma}_{u}^{T}\right]$ of the polytope defined as

$$
\left\{\begin{array}{l}
\boldsymbol{m}=\tilde{\boldsymbol{m}}+C_{u} \cdot \boldsymbol{\sigma}_{\boldsymbol{u}} \\
m_{j}=\max \left\{\tilde{m}_{j}, \alpha \cdot \operatorname{Pre}\left(p_{j}, t\right)\right\}, \quad \forall p_{j} \in \bullet^{\bullet} t \\
\boldsymbol{m}, \boldsymbol{\sigma}_{u} \geq \mathbf{0}
\end{array}\right.
$$

b) let $E=E \cup E_{i}$.

4) Remove from $E$ those vertices that are not minimal w.r.t. the firing vector.

5) Let $Y_{\min }(\mathcal{M}, t(\alpha))$ be the convex hull of $E$.

There are two main differences between Algorithms 5 and 10. Firstly, in Algorithm 10 since our goal is that of characterizing minimal e-vectors, we limit to consider those sequences of silent transitions that lead to a fluid content in the input places of $t$ that is strictly necessary to enable its firing for an amount $\alpha$. Obviously, if some of such input places already have a sufficient fluid content (that may also be greater than that strictly necessary) we keep it unaltered. To do this, we impose that for all places $p_{j} \in{ }^{\bullet} t$ it is $m_{j}=\max \left\{\tilde{m}_{j}, \alpha \cdot \operatorname{Pre}\left(p_{j}, t\right)\right\}^{3}$.

Let us also observe that the above equality can always be satisfied for all places $p_{j} \in{ }^{\bullet} t$ because of the assumptions of Proposition 9 on the unobservable subnet. In fact, if the net is backward conflict-free it may never occur that two silent transitions share the same output place. If the net is a state machine all input silent transitions of $p_{j}$ have at most one input place. Therefore, in both cases, if some place $p_{j} \in{ }^{\bullet} t$ has not a sufficient fluid content, we can always fire its silent input transitions in an amount to reach a marking equal to $\alpha \cdot \operatorname{Pre}\left(p_{j}, t\right)$.

The second difference between the two algorithms is that in Algorithm 10 we need to remove from the set of vertices obtained at Step 3 all those vertices that are not minimal w.r.t the firing vectors (see Step 4). Indeed, the set of vertices of (4) does not necessarily correspond to minimal e-vectors, but define a superset of the set of vertices relative to minimal e-vectors. As an example, let us consider the net system in Fig. 2. Let $\mathcal{M}=\left\{\left[\begin{array}{ll}\boldsymbol{m}_{0} & \mathbf{0}\end{array}\right]^{T}\right\}$. Assume $t_{1}(1)$ is observed. If we compute the vertices of (4) we also obtain a vertex corresponding to the firing of $\varepsilon_{7}$, even if $\varepsilon_{7}$ is not obviously a minimal explanation!

An easy way (even if computational demanding) to remove the non-minimal vertices is that of checking for any pair $\left[\boldsymbol{m}_{1} \boldsymbol{\sigma}_{1}\right]^{T},\left[\boldsymbol{m}_{2} \boldsymbol{\sigma}_{2}\right]^{T} \in E$ if $\min _{j=1, \ldots, n_{u}}\left(\sigma_{1, j}-\sigma_{2, j}\right) \geq 0$. If it is true, the vertex $\left[\begin{array}{ll}\boldsymbol{m}_{1} & \boldsymbol{\sigma}_{1}\end{array}\right]^{T}$ does not correspond to a minimal explanation and should be removed from $E$.

Let us finally observe, as already discussed in detail in [18] when computing the set of consistent markings, that the computational complexity of Algorithms 5 and 10 can be drastically reduced taking advantage from the structure

\footnotetext{
${ }^{3}$ Note that both $\tilde{m}_{j}$ and $\alpha \cdot \operatorname{Pre}\left(p_{j}, t\right)$ are given values, so the constrain is linear.
}

of the constraints in eq. (3) and (4). In particular, all the basis of (3) and (4) can be computed off-line because they do not depend on the current observation, thus only products of matrices need to be performed on-line.

\section{BASIS MARKINGS AND J-VECTORS}

In this section we show that under the assumptions of Proposition 9, the notions of basis markings and j-vectors we introduced for discrete PNs, can still be used in the continuous case.

A basis marking $M_{b}$ is a marking reached from $\boldsymbol{m}_{0}$ with the firing of the observed word $w$ and of all unobservable transitions whose firing is necessary to enable $w$. A jvector $y \in Y_{\min }\left(\boldsymbol{m}_{0}, w\right)$ is a firing vector of unobservable transitions whose firing is necessary to reach $M_{b}$.

Definition 11: Let $\left\langle\mathcal{N}, \boldsymbol{m}_{0}\right\rangle$ be a net system where $\mathcal{N}=$ $\langle P, T, P r e$, Post $\rangle$ and $T=T_{o} \cup T_{u}$. Let $w \in T_{o}^{*}$ be an observed word. We define

$$
\mathcal{L}(w)=\left\{\sigma \in \mathcal{L}\left(\mathcal{N}, \boldsymbol{m}_{0}\right) \mid \Pi_{o}(\sigma)=w\right\}
$$

the set of firing sequences consistent with $w \in T_{o}^{*}$.

Definition 12: Let $\left\langle\mathcal{N}, \boldsymbol{m}_{0}\right\rangle$ be a contPN system where $\mathcal{N}=\langle P, T$, Pre, Post $\rangle$ and $T=T_{o} \cup T_{u}$. Let $\sigma \in$ $\mathcal{L}\left(N, \boldsymbol{m}_{0}\right)$ be a firable sequence and $w=P_{o}(\sigma)$ the corresponding observed word. We define the set of justifications of $w$ as

$$
\begin{aligned}
\mathcal{J}(w)=\left\{\sigma_{u} \in T_{u}^{*} \mid \quad\right. & {\left[\exists \sigma \in \mathcal{L}(w): \sigma_{u}=P_{u}(\sigma)\right] \wedge } \\
& {\left[\nexists \sigma^{\prime} \in \mathcal{L}(w): \sigma_{u}^{\prime}=P_{u}\left(\sigma^{\prime}\right) \wedge\right.} \\
& \left.\left.\boldsymbol{\sigma}_{u}^{\prime} \lesseqgtr \boldsymbol{\sigma}_{u}\right]\right\} .
\end{aligned}
$$

Moreover, we define

$$
Y_{\text {min }}\left(\boldsymbol{m}_{0}, w\right)=\left\{\boldsymbol{y} \in \mathbb{R}_{\geq 0}^{n_{u}} \mid \exists \sigma_{u} \in \mathcal{J}(w): \boldsymbol{\sigma}_{u}=\boldsymbol{y}\right\}
$$

the corresponding set of $j$-vectors.

In simple words, $\mathcal{J}(w)$ is the set of sequences of unobservable transitions interleaved with $w$ whose firing enables $w$ and whose firing vector is minimal. The firing vectors of these sequences are called j-vectors.

Example 13: Let us consider again the contPN system in Fig. 1. Assume $w=t_{1}(1)$ is observed. It is easy to verify that $\sigma_{u}^{\prime}=\varepsilon_{2}(1)$ and $\sigma_{u}^{\prime \prime}=\varepsilon_{3}(1)$ are justifications of $w$, namely $\sigma_{u}^{\prime}, \sigma_{u}^{\prime \prime} \in \mathcal{J}(w)$.

This is consistent with the result already discussed in Example 8 where we claimed that $\boldsymbol{\sigma}_{u}^{\prime}, \boldsymbol{\sigma}_{u}^{\prime \prime} \in Y_{\min }\left(\boldsymbol{m}_{0}, w\right)$, where $\boldsymbol{\sigma}_{u}^{\prime}=\left[\begin{array}{ll}1 & 0\end{array}\right]^{T}, \boldsymbol{\sigma}_{u}^{\prime \prime}=\left[\begin{array}{ll}0 & 1\end{array}\right]^{T}$.

Definition 14: Let $\left\langle\mathcal{N}, \boldsymbol{m}_{0}\right\rangle$ be a net system where $\mathcal{N}=$ $\langle P, T$, Pre, Post $\rangle$ and $T=T_{o} \cup T_{u}$. Let $w$ be a given observation and $\sigma_{u} \in \mathcal{J}(w)$ be one of its minimal justifications. The marking $M_{b}=\boldsymbol{m}_{0}+C_{u} \cdot \boldsymbol{\sigma}_{u}+C \cdot \boldsymbol{\sigma}_{w}$, i.e., the marking reached firing $w$ interleaved with the minimal justification $\sigma_{u}$, is called basis marking and $\sigma_{u}$ is its $j$-vector (or justification-vector).

Example 15: Markings $\boldsymbol{m}^{\prime}$ and $\boldsymbol{m}^{\prime \prime}$ in Example 8 are basis markings for the observed word $w=t_{1}(1)$.

Obviously, because in general more than one justification exists for a word $w$ (the set $\mathcal{J}(w)$ is generally not a 
singleton), the basis marking may be not unique as well. Furthermore, two or more j-vectors may correspond to the same basis marking.

Under the assumptions of Proposition 9 the set $Y_{\min }\left(\boldsymbol{m}_{0}, w\right)$ is convex and it provides our starting point for the on-line diagnosis as discussed in the following section.

Proposition 16: Let $\left\langle\mathcal{N}, \boldsymbol{m}_{0}\right\rangle$ be a contPN. Let $w \in T_{o}^{*}$ be an observed sequence where each transition fires for a finite amount. If (A1) to (A3) hold and the $T_{u}$-induced subnet is either backward conflict-free or it is a state machine, then the set $Y_{\min }\left(\boldsymbol{m}_{0}, w\right)$ is convex.

Proof: We prove this by induction on the number of observed transitions.

(Basis step.) Let $t^{\prime}$ be the first observed transition. Assume that it has been observed for an amount $\alpha^{\prime}$. By Proposition 9 the set $Y_{\min }\left(\boldsymbol{m}_{0}, t^{\prime}\left(\alpha^{\prime}\right)\right)$ is convex.

(Inductive step.) Assume that the set $Y_{\min }\left(\boldsymbol{m}_{0}, v\right)$ is convex. We have to prove that the set $Y_{\min }\left(\boldsymbol{m}_{0}, w\right)$ is convex as well, where $w=v t(\alpha)$.

If the $T_{u}$-induced net is backward conflict-free then the result is true since the set of minimal explanations and consequently the set of basis markings are singleton [19].

Assume now that the $T_{u}$-induced net is a state machine. In this class of nets the firing vectors with minimum components corresponding to the moving of one token from any place to any other place, e.g., $p_{i} \in{ }^{\bullet} t$, have binary entries. Hence the minimal explanations have binary entries as well, thus implying that the set $Y_{\min }\left(\boldsymbol{m}_{0}, w\right)$ is convex.

An algorithm to compute $Y_{\min }\left(\boldsymbol{m}_{0}, w\right)$ can be obtained as a generalization of Algorithm 10.

Algorithm 17 (Computation of $Y_{\min }\left(\boldsymbol{m}_{0}, w\right)$ ):

1) Let $v=\varepsilon$.

2) Let $Y_{\min }\left(\boldsymbol{m}_{0}, v\right)=\left\{\left[\begin{array}{c}\boldsymbol{m} \\ \boldsymbol{y}\end{array}\right]=\left[\begin{array}{c}\boldsymbol{m}_{0} \\ \mathbf{0}\end{array}\right]\right\}$.

3) Let $t(\alpha)$ be a new observation and $w=v t(\alpha)$.

4) Compute the set of vertices $\mathcal{E}(v)$ of $Y_{\min }\left(\boldsymbol{m}_{0}, v\right)$.

5) Let $E=\emptyset$.

6) For all $\boldsymbol{e}_{i}=\left[\begin{array}{ll}\tilde{\boldsymbol{m}}^{T} & \tilde{\boldsymbol{\sigma}}_{u}^{T}\end{array}\right]^{T} \in \mathcal{E}(v)$ :

a) compute the set of vertices $E_{i}=\left[\boldsymbol{m}^{T} \boldsymbol{y}^{T}\right]^{T}$ of the polytope defined as

$$
\left\{\begin{array}{l}
\boldsymbol{m}^{\prime}=\tilde{\boldsymbol{m}}+C_{u} \cdot \boldsymbol{y} \\
m_{j}^{\prime}=\max \left\{\tilde{m}_{j}, \alpha \cdot \operatorname{Pre}\left(p_{j}, t\right)\right\}, \quad \forall p_{j} \in \bullet \cdot t \\
\boldsymbol{y}=\boldsymbol{\sigma}_{u}+\tilde{\boldsymbol{\sigma}}_{u} \\
\boldsymbol{m}=\boldsymbol{m}^{\prime}+C(\cdot, t(\alpha)) \\
\boldsymbol{m}^{\prime}, \boldsymbol{\sigma}_{u} \geq \mathbf{0}
\end{array}\right.
$$

b) let $E=E \cup E_{i}$.

7) Remove from $E$ those vertices that are not minimal W.r.t. the firing vector.

8) Let $Y_{\min }\left(\boldsymbol{m}_{0}, w\right)$ be the convex hull of $E$.

9) Let $v=w$ and goto Step 3 .

Note that, given a generic element $\boldsymbol{e}=\left[\begin{array}{ll}\boldsymbol{m}^{T} & \boldsymbol{\sigma}_{u}^{T}\end{array}\right] \in$ $Y_{\min }\left(\boldsymbol{m}_{0}, w\right)$ (not only a vertex) the first $m$ entries correspond to a basis marking and the last $n_{u}$ entries correspond

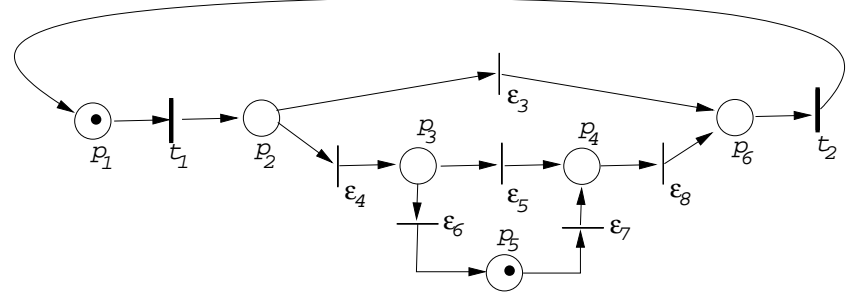

Fig. 2. The Petri net of Example 18, where $\varepsilon_{3}, \varepsilon_{4}, \varepsilon_{5}, \varepsilon_{6}, \varepsilon_{7}$ and $\varepsilon_{8}$ are not observable.

to a $j$-vector. In particular, $\boldsymbol{m}$ is the basis marking corresponding to the $j$-vector $\boldsymbol{\sigma}_{u}$.

Example 18: Let us consider the contPN system in Fig. 2 where $T_{u}=\left\{\varepsilon_{3}, \ldots, \varepsilon_{8}\right\}$. The $T_{u}$-induced subnet is a state machine.

Let $w=t_{1}(1) t_{2}(1) t_{2}(1)$ be the current observation. The set of vertices computed at the different iterations of Algorithm 17 are summarized in the following items.

- $w=\varepsilon$, i.e., $w$ is the empty word.

$Y_{\min }\left(\boldsymbol{m}_{0}, \varepsilon\right)=\left\{\left[\begin{array}{lllllllllllll}1 & 0 & 0 & 0 & 1 & 0 & 0 & 0 & 0 & 0 & 0 & 0\end{array}\right]^{T}\right\}$ where the first six entries correspond to the basis marking (that coincides with $\boldsymbol{m}_{0}$ ) and the last six entries correspond to the minimal $\mathrm{j}$-vector that is initially null by definition.

- $w=t_{1}(1)$. $Y_{\text {min }}\left(\boldsymbol{m}_{0}, t_{1}(1)\right)=\left\{\left[\begin{array}{llllll|llllll}0 & 1 & 0 & 0 & 1 & 0 & 0 & 0 & 0 & 0 & 0 & 0\end{array}\right]^{T}\right\}$. The firing of $t_{1}(1)$ is enabled at the initial marking thus we only have one $\mathrm{j}$-vector that coincides with the null vector, and the basis marking is obtained from the initial marking (the basis marking at the previous iteration) firing $t_{1}(1)$.

- $w=t_{1}(1) t_{2}(1)$.

The set of vertices obtained at Step 6 of Algorithm 17 is given by the the following elements:

1) $e_{1}=\left[\begin{array}{llllll|llllll}1 & 0 & 0 & 0 & 1 & 0 & 0 & 1 & 1 & 0 & 0 & 1\end{array}\right]^{T}$,

2) $e_{2}=\left[\begin{array}{llllll|llllll}1 & 1 & 0 & 0 & 0 & 0 & 0 & 0 & 0 & 0 & 1 & 1\end{array}\right]^{T}$,

3) $\boldsymbol{e}_{3}=\left[\begin{array}{llllll|llllll}1 & 0 & 0 & 0 & 1 & 0 & 1 & 0 & 0 & 0 & 0 & 0\end{array}\right]^{T}$,

4) $e_{4}=\left[\begin{array}{llllll|llllll}1 & 0 & 0 & 1 & 0 & 0 & 1 & 0 & 0 & 0 & 1 & 0\end{array}\right]^{T}$, that correspond respectively to the justifications $\sigma_{1}=$ $\varepsilon_{4} \varepsilon_{5} \varepsilon_{8}, \sigma_{2}=\varepsilon_{7} \varepsilon_{8}, \sigma_{3}=\varepsilon_{3}, \sigma_{4}=\varepsilon_{3} \varepsilon_{7}$.

However, at Step 7 the last element $\boldsymbol{e}_{4}$ is removed because it does not correspond to a minimal $\mathrm{j}$-vector. Indeed, the firing of $\varepsilon_{7}$ is not necessary to enable $t_{2}(1)$ at the previous basis marking if $\varepsilon_{3}$ has already fired.

- $w=t_{1}(1) t_{2}(1) t_{2}(1)$.

The set of vertices obtained at Step 6 of Algorithm 17 is given by the following elements:

1) $e_{1}^{\prime}=\left[\begin{array}{llllll|llllll}2 & 0 & 0 & 0 & 0 & 0 & 0 & 1 & 1 & 0 & 1 & 2\end{array}\right]^{T}$,

2) $\boldsymbol{e}_{2}^{\prime}=\left[\begin{array}{llllll|llllll}2 & 0 & 0 & 0 & 0 & 0 & 1 & 0 & 0 & 0 & 1 & 1\end{array}\right]^{T}$,

that all correspond to minimal j-vectors, thus none of them is removed at Step 7.

\section{FAUlt Diagnoser DESIGN}

Let us consider a system modeled as a contPN whose transitions may either be observable or unobservable $(T=$ $T_{o} \cup T_{u}$ ). Assume that a certain number of anomalous (or 
fault) behaviors may occur in the system. The occurrence of a fault behavior corresponds to the firing of an unobservable transition, but there may also be other transitions that are unobservable as well, but whose firing corresponds to regular behaviors. Then, assume that fault behaviors may be divided into $r$ main classes (fault classes), and we are not interested in distinguishing among fault events in the same class. This can be easily modeled in PN terms assuming that the set of unobservable transitions is partitioned into two subsets, namely $T_{u}=T_{f} \cup T_{\text {reg }}$, where $T_{f}$ includes all fault transitions and $T_{\text {reg }}$ includes all transitions relative to unobservable but regular events. The set $T_{f}$ is further partitioned into $r$ subsets, namely, $T_{f}=T_{f}^{1} \cup T_{f}^{2} \cup \ldots \cup T_{f}^{r}$ where all transitions in the same subset correspond to the same fault class. We will say that the $i$-th fault has occurred when a transition in $T_{f}^{i}$ has fired.

We now provide the definition of diagnoser and diagnosis state that we first introduced in [13].

Definition 19: A diagnoser is a function $\Delta: T_{o}^{*} \times$ $\left\{T_{f}^{1}, T_{f}^{2}, \ldots, T_{f}^{r}\right\} \rightarrow\{0,1,2,3\}$ that associates to each observation $w$ and to each fault class $T_{f}^{i}, i=1, \ldots, r$, a diagnosis state.

- $\Delta\left(w, T_{f}^{i}\right)=0$ if for all $\sigma \in \mathcal{L}(w)$ and for all $t_{f} \in T_{f}^{i}$ it holds $t_{f} \notin \sigma$.

In such a case the $i$ th fault cannot have occurred, because none of the firing sequences consistent with the observation contains fault transitions of class $i$.

- $\Delta\left(w, T_{f}^{i}\right)=1$ if:

(i) there exist $\sigma \in \mathcal{L}(w)$ and $t_{f} \in T_{f}^{i}$ such that $t_{f} \in \sigma$ but

(ii) for all $\sigma \in \mathcal{J}(w)$ and for all $t_{f} \in T_{f}^{i}$ it holds that $t_{f} \notin \sigma$.

In such a case a fault transition of class $i$ may have occurred but is not contained in any justification of $w$.

- $\Delta\left(w, T_{f}^{i}\right)=2$ if there exist $\sigma, \sigma^{\prime} \in \mathcal{J}(w)$ such that:

(i) there exists $t_{f} \in T_{f}^{i}$ such that $t_{f} \in \sigma$;

(ii) for all $t_{f} \in T_{f}^{i}, t_{f} \notin \sigma^{\prime}$.

In such a case a fault transition of class $i$ is contained in one (but not in all) justification of $w$.

- $\Delta\left(w, T_{f}^{i}\right)=3$ if for all $\sigma \in \mathcal{L}(w)$ there exists $t_{f} \in T_{f}^{i}$ such that $t_{f} \in \sigma$.

In such a case the $i$ th fault must have occurred, because all firable sequences consistent with the observation contain at least one fault transition of class $i$.

The diagnosis states 1 and 2 correspond both to cases in which a fault may have occurred but has not necessarily occurred. The main reason to distinguish between them is the following. In the state 1 the observed behavior does not suggest that a fault has occurred because all minimal sequences leading to $w$ are fault free. On the contrary, in the state 2 at least one of the justifications of the observed behavior contains one transition in the class.

Example 20: Consider the contPN system in Fig. 2 already examined in Example 18. Assume two fault classes: $T_{f}^{1}=\left\{\varepsilon_{3}\right\}$ and $T_{f}^{2}=\left\{\varepsilon_{6}, \varepsilon_{7}\right\}$.

- Let $w=\varepsilon$. The set of justifications is empty, thus the diagnosis states may either be equal to 0 or 1 . Now, since $\varepsilon_{3}$ is not enabled at the initial marking it is $\Delta\left(w, T_{f}^{1}\right)=0$. On the contrary, transition $\varepsilon_{7}$ is enabled at the initial marking thus $\Delta\left(w, T_{f}^{2}\right)=1$.

- Let $w=t_{1}(1)$. The set of justifications at $w$ only contains the empty word because $t_{1}(1)$ is enabled at the initial marking. However, both $\varepsilon_{3}$ and $\varepsilon_{7}$ can fire after the occurrence of $t_{1}(1)$. Thus, $\Delta\left(w, T_{f}^{1}\right)=\Delta\left(w, T_{f}^{2}\right)=$ 1.

- Let $w=t_{1}(1) t_{2}(1)$. Looking at the set of minimal $\mathbf{j}$ vectors computed in Example 18 it is easy to observe that for both fault classes, we have some justification that contains at least one transition in the class, but we also have justifications that contain none of them, thus $\Delta\left(w, T_{f}^{1}\right)=\Delta\left(w, T_{f}^{2}\right)=2$.

- Let $w=t_{1}(1) t_{2}(1) t_{2}(1)$. Always looking at the set of minimal j-vectors computed in Example 18 we argue that $\Delta\left(w, T_{f}^{1}\right)=2$ and $\Delta\left(w, T_{f}^{2}\right)=3$. In fact, while transition $\varepsilon_{3}$ is contained in only one of the two justifications at $w$, transition $\varepsilon_{7}$ is contained in both of them.

The on-line computation of the sets $\mathcal{L}(w)$ and $\mathcal{J}(w)$ may be computational demanding in large scale systems, thus in the following we suggest an alternative procedure to compute diagnosis states that is based on the knowledge of the the set $Y_{\min }\left(\boldsymbol{m}_{0}, w\right)$. In particular, such a procedure well applies to all cases in which the set $Y_{\min }\left(\boldsymbol{m}_{0}, w\right)$ is convex. Thus for sure it well applies to net systems whose unobservable subnet is either backward conflict-free or a state machine.

Proposition 21: Consider an observed word $w \in T_{o}^{*}$. Let

$$
\begin{aligned}
& \left\{\begin{array}{l}
l_{i}=\min \sum_{t_{j} \in T_{f}^{i}} \sigma_{u}\left(t_{j}\right) \\
\text { s.t. } \\
{\left[\begin{array}{l}
\boldsymbol{m} \\
\boldsymbol{\sigma}_{u}
\end{array}\right] \in Y_{\min }\left(\boldsymbol{m}_{0}, w\right)}
\end{array}\right. \\
& \left\{\begin{array}{l}
u_{i}=\max \sum_{t_{j} \in T_{f}^{i}} \sigma_{u}\left(t_{j}\right) \\
\text { s.t. } \\
{\left[\begin{array}{l}
\boldsymbol{m} \\
\boldsymbol{\sigma}_{u}
\end{array}\right] \in Y_{\min }\left(\boldsymbol{m}_{0}, w\right)}
\end{array}\right. \\
& \left\{\begin{array}{l}
\bar{u}_{i}=\max \sum_{t_{j} \in T_{f}^{i}} \bar{\sigma}_{u}\left(t_{j}\right) \\
\text { s.t. } \\
{\left[\begin{array}{l}
\boldsymbol{m} \\
\boldsymbol{\sigma}_{u}
\end{array}\right] \in Y_{\min }\left(\boldsymbol{m}_{0}, w\right)} \\
\boldsymbol{m}+C_{u} \cdot \overline{\boldsymbol{\sigma}}_{u} \geq \mathbf{0}
\end{array}\right.
\end{aligned}
$$

It holds:

$$
\begin{aligned}
& \Delta\left(w, T_{f}^{i}\right)=0 \quad \Leftrightarrow \quad u_{i}=\bar{u}_{i}=0 \\
& \Delta\left(w, T_{f}^{i}\right)=1 \quad \Leftrightarrow \quad u_{i}=0, \bar{u}_{i}>0 \\
& \Delta\left(w, T_{f}^{i}\right)=2 \quad \Leftrightarrow \quad l_{i}=0, u_{i}>0 \\
& \Delta\left(w, T_{f}^{i}\right)=3 \quad \Leftrightarrow \quad l_{i}>0
\end{aligned}
$$

Proof: It follows from Definitions 12 and 19. 
If $u_{i}=\bar{u}_{i}=0$ it means that none of the justifications contains transitions in $T_{f}^{i}$ and no transition in $T_{f}^{i}$ may also have fired after any of such justifications. But by Definition 19 this is exactly what corresponds to diagnosis state 0 . Moreover, if $u_{i} \neq 0$ it means that at least one justification contains at least one transition in the $i$ th class, thus the diagnosis state cannot be 0 . Finally, if $u_{i} \neq 0$ but $\bar{u}_{i} \neq 0$ it means that at least one transition in the $i$ th fault class may have fired after the firing of any of the justifications at $w$. In such a case the diagnosis state is $>0$.

If $u_{i}=0$ and $\bar{u}_{i}>0$ it means that none of the justifications contains transitions in $T_{f}^{i}$ but at least one transition in $T_{f}^{i}$ may have fired after a basis marking, thus the diagnosis state is 1 . Obviously, the diagnosis state cannot be equal to 1 if either $u_{i} \neq 0$ or $\bar{u}_{i}=0$.

If $l_{i}=0$ and $u_{i}>0$ it means that at least one of the justifications at $w$ contains some transition in the $i$ th class, that is by definition the case of diagnosis state equal to 2 . If any of such conditions is violated the diagnosis state cannot be equal to 2 .

Finally, if $l_{i}>0$ then all the justifications at $w$ contain at least one transition in $T_{f}^{i}$, i.e., all words consistent with the actual observation contain a transition in the $i$ th class, that means that some fault in the $i$ th class has occurred for sure. This corresponds by Definition 19 to diagnosis state equal to 3 . If $l_{i}=0$ it means that some justification contains no transition in $T_{f}^{i}$ thus the diagnosis state is $<3$.

Example 22: Consider the contPN system in Fig. 2 already examined in Examples 18 and 20. Assume two fault classes: $T_{f}^{1}=\left\{\varepsilon_{3}\right\}$ and $T_{f}^{2}=\left\{\varepsilon_{6}, \varepsilon_{7}\right\}$. We compute the diagnosis states after certain observations solving the LPPs in Proposition 21. Obviously such results are consistent with those obtained in Example 20, based on the definition of diagnosis states.

- $w=\varepsilon$.

1) $l_{1}=u_{1}=\bar{u}_{1}=0 \Longrightarrow \Delta\left(w, T_{f}^{1}\right)=0$;

2) $l_{2}=u_{2}=0$ and $\bar{u}_{2}=1 \Longrightarrow \Delta\left(w, T_{f}^{2}\right)=1$.

- $w=t_{1}(1)$.

1) $l_{1}=u_{1}=0$ and $\bar{u}_{1}=1 \Longrightarrow \Delta\left(w, T_{f}^{1}\right)=1$;

2) $l_{2}=u_{2}=0$ and $\bar{u}_{2}=3 \Longrightarrow \Delta\left(w, T_{f}^{2}\right)=1$.

- $w=t_{1}(1) t_{2}(1)$.

1) $l_{1}=0, u_{1}=1$ and $\bar{u}_{1}=1 \Longrightarrow \Delta\left(w, T_{f}^{1}\right)=2$;

2) $l_{2}=0, u_{2}=1$ and $\bar{u}_{2}=2 \Longrightarrow \Delta\left(w, T_{f}^{2}\right)=2$.

- $w=t_{1}(1) t_{2}(1) t_{2}(1)$.

1) $l_{1}=0, u_{1}=1$ and $\bar{u}_{1}=0 \Longrightarrow \Delta\left(w, T_{f}^{1}\right)=2$;

2) $l_{2}=u_{2}=1$ and $\bar{u}_{2}=0 \Longrightarrow \Delta\left(w, T_{f}^{2}\right)=3$.

\section{CONCLUSIONS AND FUTURE WORK}

In this paper we presented an approach for the design of a fault diagnoser for systems modeled by untimed contPNs. A certain number of transitions, including all the fault transitions, are assumed to be unobservable. Fault transitions are partitioned into different fault classes and the diagnoser assigns four different states (corresponding to different degrees of alarm) to each fault class based on the actual observation. We proved that under certain assumptions on the unobservable subnet the set of $j$-vectors is convex thus the diagnosis state may be easily computed solving a LPP.

We plan to extend this work in several directions. Firstly, we want to characterize how the number of vertices of the polytope defining the set of minimal explanations changes with the observed word. Secondly, we plan to extend the diagnosis approach to more general classes of Petri nets for which the set of minimal explanations is not convex. Finally, we will try to derive some criteria for diagnosability, namely some criteria to establish a priori if the fault occurrences can be reconstructed after a finite amount of observations.

\section{REFERENCES}

[1] R. Boel and J. van Schuppen, "Decentralized failure diagnosis for discrete-event systems with costly communication between diagnosers," in Proc. WODES'02: 6th Work. on Discrete Event Systems (Zaragoza, Spain), Oct. 2002, pp. 175-181.

[2] R. Debouk, S. Lafortune, and D. Teneketzis, "Coordinated decentralized protocols for failure diagnosis of discrete-event systems," Discrete Events Dynamical Systems, vol. 20, pp. 33-79, 2000.

[3] S. H. Zad, R. Kwong, and W. Wonham, "Fault diagnosis in discreteevent systems: framework and model reduction," IEEE Trans. Automatic Control, vol. 48 (7), pp. 1199-1212, 2003.

[4] S. Jiang and R. Kumar, "Failure diagnosis of discrete-event systems with linear-time temporal logic specifications," IEEE Trans. Automatic Control, vol. 49, no. 6, pp. 934-945, Jun. 2004.

[5] J. Lunze and J. Schroder, "Sensor and actuator fault diagnosis of systems with discrete inputs and outputs," IEEE Trans. Systems, Man and Cybernetics, Part B, vol. 34, no. 3, Apr. 2004.

[6] M. Sampath and S. Lafortune, "Active diagnosis of discrete-event systems," IEEE Trans. Automatic Control, vol. 43, pp. 908-929, 1998.

[7] M. Sampath, R. Sengupta, S. Lafortune, K. Sinnamohideen, and D. Teneketzis, "Diagnosability of discrete-event systems," IEEE Trans. Automatic Control, vol. 40 (9), pp. 1555-1575, 1995.

[8] T. Ushio, L. Onishi, and K. Okuda, "Fault detection based on Petri net models with faulty behaviors," in Proc. SMC'98: IEEE Int. Conf. on Systems, Man, and Cybernetics (San Diego, CA, USA), Oct. 1998, pp. $113-118$.

[9] A. Aghasaryan, E. Fabre, A. Benveniste, R. Boubour, and C. Jard, "Fault detection and diagnosis in distributed systems: an approach by partially stochastic Petri nets," Discrete Events Dynamical Systems, vol. 8, pp. 203-231, Jun. 1998.

[10] A. Benveniste, E. Fabre, S. Haar, and C. Jard, "Diagnosis of asynchronous discrete event systems, a net unfolding approach," IEEE Trans. Automatic Control, vol. 48, no. 5, pp. 714-727, May 2003.

[11] G. Jiroveanu and R. Boel, "A distributed approach for fault detection and diagnosis based on time Petri nets," Mathematics and Computers in Simulation, vol. 70, pp. 287-313, 2006.

[12] A. Giua and C. Seatzu, "Fault detection for discrete event systems using Petri nets with unobservable transitions," in Proc. 44th IEEE Conf. on Decision and Control, Dec. 2005, pp. 6323-6328.

[13] M. P. Cabasino, A. Giua, and C. Seatzu, "Fault detection for discrete event systems using Petri nets with unobservable transitions," Automatica, (preliminary accepted).

[14] H. Alla and R. David, "Continuous and hybrid Petri nets," Journal of Circuits, Systems, and Computers, vol. 8, no. 1, pp. 159-188, 1998.

[15] M. Silva and L. Recalde, "On fluidification of Petri net models: from discrete to hybrid and continuous models," Annual Reviews in Control, vol. 28, no. 2, pp. 253-266, 2004.

[16] L. Recalde, E. Teruel, and M. Silva, "Autonomous continuous P/T systems," in Application and Theory of Petri Nets 1999, ser. Lecture Notes in Computer Science, J. K. S. Donatelli, Ed., vol. 1639. Springer, 1999, pp. 107-126.

[17] T. Murata, "Petri nets: Properties, analysis and applications," Proceedings of the IEEE, vol. 77, no. 4, pp. 541-580, 1989.

[18] C. Mahulea, C. Seatzu, M. P. Cabasino, L. Recalde, and M. Silva, "Observer Design for Untimed Continuous Petri Nets," in 2009 American Control Conference, St. Louis, Missouri, USA, June 2009.

[19] D. Corona, A. Giua, and C. Seatzu, "Marking estimation of Petri nets with silent transitions," IEEE Transaction on Automatic Control, vol. 52, no. 9, pp. 1695 - 1699, 2007. 